\title{
-
}

\section{USP2-45 Represses Aldosterone Mediated Responses by Decreasing Mineralocorticoid Receptor Availability}

\author{
Nourdine Faresse ${ }^{a, b} \quad$ Anne Debonneville Olivier Staub $^{b}$ \\ aInstitute of Anatomy, University of Zurich, Zurich, Switzerland; bepartment of Pharmacology and \\ Toxicology, University of Lausanne, Lausanne, Switzerland
}

\section{Key Words}

Aldosterone $\cdot$ Ubiquitin $•$ Proteasome $\cdot$ Nuclear receptor $•$ Post-translational modification

\begin{abstract}
Background/Aims: Ligand activation of the mineralocorticoid receptor (MR) induces several post-translational modifications (PTMs). Among the different PTMs, MR is known to be dynamically ubiquitylated with impact on its stability and transcriptional activity. Previously, we have shown that MR is monoubiquitylated at the basal state and that aldosterone stimulation induces monoubiquitylation removal prompting polyubiquitin-dependent destabilization of the receptor and proteasomal degradation. This study investigated the role of the aldosterone induced ubiquitin-specific protease USP2-45 on the ubiquitylation state of MR. Methods: Renal epithelial cells M1 were co-transfected with MR with or without wild-type or inactive USP2-45. The association of MR with USP2-45 or TSG101 as well as MR ubiquitylation state were determined by immunoprecipitation and immunoblotting. MR transcriptional activity was assessed via a luciferase reporter gene. Results: We show that USP2-45 is able to bind $M R$ and, similarly to aldosterone, induce MR monoubiquitylation removal, disruption of MR/ TSG101 association and destabilization of MR at protein level. Conclusion: This study provides a novel role for USP2-45 by playing a pivotal role in the regulation of the ubiquitylation state of $\mathrm{MR}$ and reveals the existence of a negative feedback loop for limiting the aldosterone induced response.
\end{abstract}

Copyright (C) 2013 S. Karger AG, Basel

\section{Introduction}

Aldosterone is a mineralocorticoid hormone secreted by the zona glomerulosa of the adrenal cortex. This hormone mediates its effects through binding to the mineralocorticoid receptor (MR or NR3C2), which is expressed in a wide range of tissues and belongs to the steroid/thyroid hormone receptor superfamily [1, 2]. 
The importance of MR in $\mathrm{Na}^{+}$homeostasis and blood pressure control was demonstrated by either loss-of-function mutations in the MR encoding gene, causing autosomal dominant pseudohypoaldosteronism type 1 (adPHA1) [3], or gain-of-function mutations leading to a severe inherited form of early-onset hypertension [4]. In addition to its involvement in hypertension, MR signaling contributes to the appearance of cardiac alterations, heart failure or diabetes [5]. Clinical studies such as RALES, EPHESUS or 4E revealed that MR antagonists decrease organ damage, hospitalization of patients with hypertension and heart failure [6-8]. These clinical observations highlight the importance of MR in cardiovascular pathophysiology and the necessity to characterize the mechanisms of MR activation and regulation.

Several post-translational modifications (PTMs) on the MR have been described, for the majority after ligand binding [9]. Among these different PTMs, phosphorylation appears to play a major role in modulating the intrinsic MR function. Recently, we have shown that aldosterone induces MR phosphorylation at different residues via ERK1/2 and these modifications are critical for the regulation of the MR ubiquitylation state [10]. Indeed, we have found that MR is monoubiquitylated in its basal state and this status is sustained by the tumor suppressor gene 101 (TSG101). Phosphorylation of the receptor leads to disruption of MR/TSG101 association and monoubiquitin removal, leading to polyubiquitin-dependent destabilization of MR via proteasomal degradation [10].

Protein ubiquitylation results from the sequential action of 3 enzymes: the ubiquitinactivating enzyme E1, an ubiquitin-conjugating enzyme E2 and an ubiquitin-protein ligase E3 [11]. Ubiquitin is covalently conjugated to the $\varepsilon$-amino group of lysine residues; such lysines can be modified either by a single ubiquitin (referred to as monoubiquitylation) or by polyubiquitin chains linked via internal lysines [11]. Similarly to phosphorylation, ubiquitylation is reversible due to the action of deubiquitinases (DUBs, also referred to as deubiquitylating enzymes) that catalytically cleave the ubiquitin from their substrates. There are approximately 80 DUBs in the human genome that are classified into different protein families, including Ubiquitin-Specific Proteases (USPs), Ubiquitin-Carboxyterminal Hydrolases (UCHs), Ovarian Tumor Proteases (OTUs), Josephins, and JAB1/MPN/MOV34 Metalloenzymes (JAMMs; also known as MPN+ and often referred to as JAMM/MPN+) [12]. The USP family is the largest one with close to 60 enzymes $[12,13]$ that are characterized by the presence of an USP catalytic domain containing essential cysteine and histidine boxes, and varying $\mathrm{N}$ - and $\mathrm{C}$-terminal extensions. USP2 was originally identified in rat testis, where it is highly expressed [14], and exists in 2 isoforms, now referred to as USP2-45 and USP269 , according to their apparent molecular weight [15]. It was then found that USP-45 is an aldosterone early-induced protein, which is able to bind to and deubiquitylate $\mathrm{ENaC}$, leading to elevated ENaC activity [16].

In the context of aldosterone signaling, we described MR as a monoubiquitylated protein in the basal state [10], which upon stimulation by aldosterone becomes deubiquitylated, and subsequently polyubiquitylated and degraded. The DUB involved in the monoubiquitylation removal after aldosterone stimulation remained unknown. Based on the observation that aldosterone induces the expression of USP2-45 [16], and plays a role in the regulation of $\mathrm{ENaC}$ [17], we hypothesized that USP2-45 could be responsible for aldosterone mediated deubiquitylation of MR. We provide now evidence that USP2-45 is a MR partner at the basal state, which is involved in receptor deubiquitylation. We also describe the antagonizing effect of USP2-45 on aldosterone induced transactivation, by inducing destabilization and degradation of MR via the proteasome.

\section{Materials and Methods}

Cells culture and transfection

The cortical collecting duct cells M1 (ATCC) were cultured in medium containing DMEM-F12 and 5\% fetal calf serum. These cells were transfected with Lipofectamine according the manufacturer's protocol (Invitrogen). 
Faresse/Debonneville/Staub: Deubiquitylation Induces MR Degradation

\section{Plasmids and Antibodies}

Expression vectors: pCMV4-hMR , pcDNA3.1 Stag-hMR wt, Stag-USP2-45 wild type and C67A were generated by PCR and cloned into pcDNA3 [10]. The HA-ubiquitin WT were kindly provided by P. Shaw (University of Lausanne, Switzerland). Expression vector encoding shUSP2-45, oligonucleotides containing sequences from mouse USP2-45 were inserted into the expression vector pSUPER vector according to the manufacturer's instructions (Invitrogen). The following antibodies used were: anti-MR (1/100 [18] for western-blot, anti-USP2 [17] anti-MR (Santa-Cruz; sc-11412) for immunoprecipitation, anti-actin (1/1000 Sigma; a-2066), anti-Stag (1/5000 Novagen; 71549-3), anti-TSG101 (1/3000 Santa Cruz; sc-7964), antiUbiquitin FK2 (1/1000 Enzo; PW8810), anti-HA (1/500 Santa Cruz; sc-7392).

Tissue lysate

USP2 knock-out mice were purchased from Deltagen. The proteins were extracted by homogenizing the kidneys or testis using Polytron in extraction solution ( $250 \mathrm{mM}$ sucrose, $150 \mathrm{mM} \mathrm{NaCl}, 30 \mathrm{mM}$ Tris$\mathrm{HCl} \mathrm{pH} \mathrm{7.5,} \mathrm{and} \mathrm{Complete} \mathrm{protease} \mathrm{inhibitor} \mathrm{cocktail} 1$ tablet/20 ml; Roche). Prior to use $1 \mathrm{mM} \mathrm{DTT,} 100$ $\mathrm{mM}$ sodium fluoride, and $10 \mathrm{mM}$ disodium pyrophosphate were added to the extraction solution. After centrifugation at $15000 \mathrm{~g}$ for $15 \mathrm{~min}$ at $4^{\circ} \mathrm{C}$, supernatants were collected and assayed for total protein.

Immunoblotting and immunoprecipitation

Procedures for immunoprecipitation and immunoblotting were as described previously [19].

Transcriptional reporter assay

M1 cells were seeded into 24-well plates then co-transfected by Lipofectamine with the reporter gene MMTV-luc (kindly provided by P.J Fuller [20]), the constitutive luciferase vector coding for Renilla and the indicated plasmids. After 30h, cells were exposed to aldosterone (30 nM) for 16h. After hormone treatment, cells were washed twice with phosphate-buffered saline (PBS) and lysed with $100 \mu \mathrm{l}$ of passive lysis buffer (Promega). Luciferase activity was determined by using a luciferase kit (Promega). Luciferase activity was normalized for constitutive Renilla luciferase.

Real-time quantitative PCR

Total RNA of transfected cells was extracted using the RNAquous Kit (Ambion). RNA (1 $\mu \mathrm{g})$ was reverse-transcribed using SuperscriptII reverse transcriptase (Invitrogen) and $1 \mu \mathrm{g}$ of random hexamer primers (Invitrogen) in a total volume of $20 \mu \mathrm{l}$. Quantitative real-time PCR was performed in replicate for each sample using the Applied Biosystems 7500 Fast Real-Time PCR System, the TaqMan Gene Expression Assays (Applied Biosystems) for MR (Mm01241596_m1) and GAPDH (Mm99999915_g1) as housekeeping gene, and the TaqMan Universal PCR Master Mix (Applied Biosystems). Diluted reverse-transcribed samples (total RNA of $5 \mathrm{ng}$ ) were amplified in a final volume of $20 \mu \mathrm{l}$. The amount of MR mRNA was normalized to GAPDH mRNA expression.

\section{Results}

USP2-45 interacts with the non-activated MR

The ubiquitin-specific protease USP2-45 was previously described as an early aldosterone-induced gene involved in the regulation of $\mathrm{Na}^{+}$reabsorption by ENaC [16]. Given that aldosterone promotes removal of MR monoubiquitylation, we tested whether USP2-45 is involved in this mechanism. First, we investigated whether USP2-45 interacts with MR in renal epithelial cells. To address this hypothesis, we co-transfected M1 cells with MR and S-tagged USP2-45. We observed that when MR and S-tagged USP2-45 were co-expressed, the two proteins co-immunoprecipitated either with anti MR or anti-Stag antibody (Fig. 1A). Moreover, we found that the catalytically inactive mutant USP2-45 CA (where the cysteine 67 was substituted to an alanine) bound MR in a manner similar to wt USP2-45. We then tested if aldosterone was affecting this interaction and found that it was not influenced by treatment with $30 \mathrm{nM}$ aldosterone during 3 hours (Fig. 1B). To map the USP2-45 domain responsible for this interaction, we made use of different USP2-45 deletion mutants co-transfected with 
Fig. 1. USP2-45 binds to MR. (A and B) M1 cells were co-transfected with MR and wt or catalytically inactive (CA) S-tagged USP2-45. (B) 3 hours before lysis, cells were treated or not with $30 \mathrm{nM}$ aldosterone. (A and B) Cell lysates were immunoprecipitated with anti-Stag or anti-MR, and analyzed by SDS-PAGE/Western-blotting using anti-MR or anti-Stag antibodies. The expression of proteins under investigation was determined by direct immunoblotting of lysates and actin as a loading control. (C) M1 cells were co-transfected with MR and different fragments of S-tagged USP245. Cell lysates were immunoprecipitated with anti-Stag and analyzed by SDS-PAGE/ Western-blotting using anti-MR or anti-Stag antibodies. The total MR expression was determined by direct immunoblotting and actin as a loading control.

MR. Immunoprecipitation experiments indicated that the MR-binding domain mapped to the catalytic carboxy-terminal region of USP2-45 (Fig. 1C). These observations suggest that MR bind the catalytic domain of USP2-45 independently of its activity.

\section{USP2-45 removes MR monoubiquitylation and disrupts MR/TSG101 association}

To assess the effect of USP2-45 on MR monoubiquitylation, we co-transfected MR with S-tagged wild-type USP2-45 or mutant USP2-45 CA. Immunoprecipitation experiments showed that MR monoubiquitylation was undetectable on immunoblots in presence of wild-type USP2-45, but not the catalytic inactive mutant (Fig. 2A). This observation suggested that catalytic activity of USP2-45 was crucial for MR monoubiquitylation removal. We have previously shown that TSG101 binds to the non-activated MR and promotes its monoubiquitylation [10]. Given that USP2-45 was responsible of MR monoubiquitylation removal, we tested whether USP2-45 interferes with MR/TSG101 association. We found 


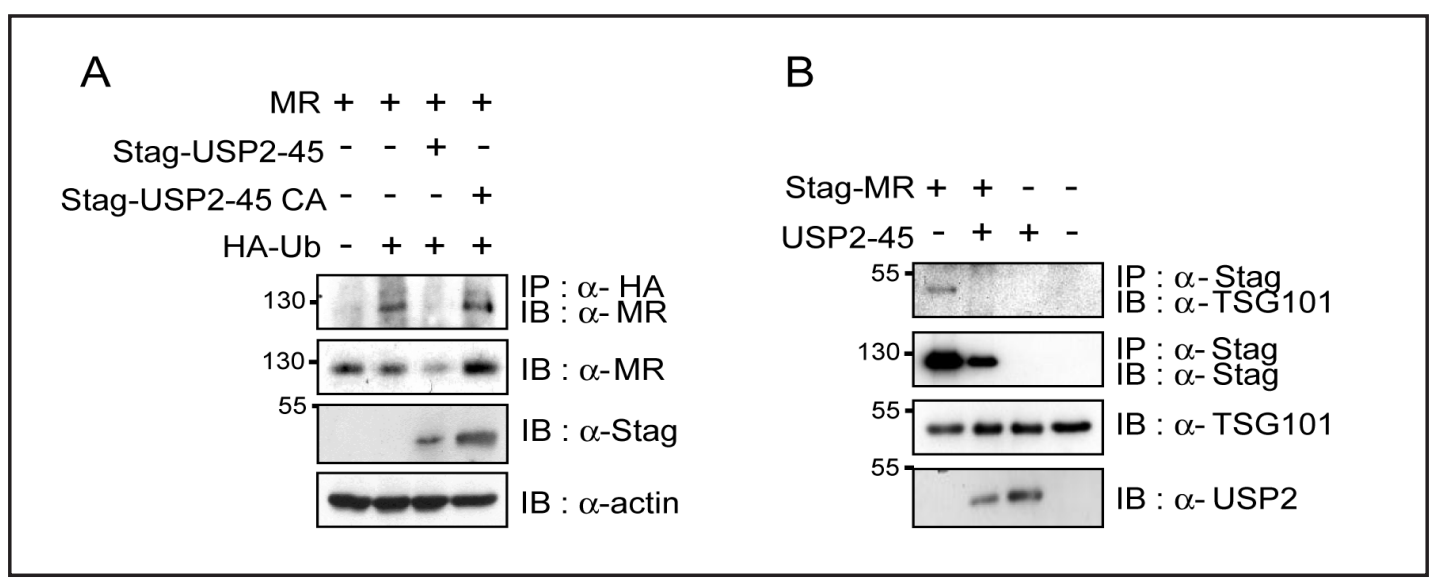

Fig. 2. USP2-45 removes MR monoubiquitylation. (A) M1 cells were co-transfected with MR, HA-Ub and Stag-USP2-45 wt or CA. Cell lysates were immunoprecipitated with anti-HA and blotted with antiMR. The expression of MR and USP2-45 was determined by direct immunoblotting of lysates and actin as a loading control. (B) M1 cells were co-transfected with S-tagged MR and USP2-45. Cell lysates were immunoprecipitated with anti-Stag and analyzed by SDS-PAGE/Western-blotting using anti-TSG101 or antiStag antibodies. The expression of proteins under investigation was determined by direct immunoblotting and actin as a loading control.

that endogenous TSG101 precipitated with MR and overexpression of USP2-45 dramatically reduced this association (Fig. 2B). This confirms that TSG101 binds to the monoubiquitylated MR and, as compared to USP2-45, has an opposite role to USP2-45 on MR ubiquitylation state.

\section{Monoubiquitylation removal by USP2-45 decreases MR protein stability}

In addition to inducing monoubiquitylation removal and TSG101 dissociation, we found that overexpression of USP2-45 decreased MR expression level (Fig. 1). These observations were confirmed by co-transfection of MR with increasing doses of USP2-45. As shown in Figure 3A, MR expression was decreased by USP2-45 in a dose-dependent manner. Such decrease in MR expression required the catalytic activity of USP2-45, since the USP2-45 CA mutant did not alter MR expression levels (Fig. 3B). Furthermore, the effect of USP2-45 on MR was specific for this short isoform of the Usp2 gene since the long isoform USP2-69, which is not induced by aldosterone, had no effect on MR (Fig. 3C). To provide further evidence to our hypothesis, we knocked down endogenous USP2-45 by RNA interference. Initially, we validated the knock-down efficiency of the designed small-interference RNA (siRNA) by cotransfecting USP2-45 with two siRNA targeted against USP2-45 (siUSP2a and shUSP2b) or with a siRNA control. As expected, USP2-45 was not detectable when it was co-transfected with both siUSP2-45, suggesting an efficient knockdown of USP2-45 protein (Fig. 3D). Using these siRNA, we found that both siUSP2-45 a and b, but not the siControl, induced higher MR expression levels (Fig. 3E). To determine the physiological relevance of our observations, we evaluated MR expression in vivo in mice deficient for the usp2 gene. As shown in Figure 3F, MR expression in the kidney was 2,8 fold higher in USP2 knock-out (KO) mice than in wildtype. The same difference in MR expression was found in testis, another tissue expressing both USP2-45 and MR (Fig. 3F).

Based on our findings, we postulated that USP2-45 acts in a similar manner to aldosterone, by removing the MR monoubiquitylation and inducing the receptor degradation as described previously [10]. To test this, we evaluated the effect of USP2-45 on MR mRNA level. As shown in Fig. 4A, co-transfection of wild-type or catalytically inactive USP2-45 mutant with MR did not affect MR mRNA levels, suggesting that USP2-45 did not affect MR mRNA stability. Then, we estimated the half-life of MR protein in the presence or absence of USP2-45. We found that overexpression of USP2-45 significantly reduced the receptor half- 
A

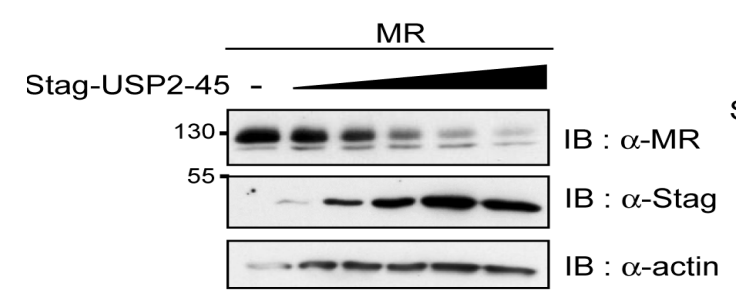

C

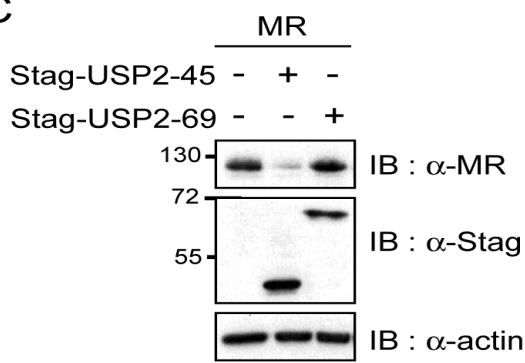

E

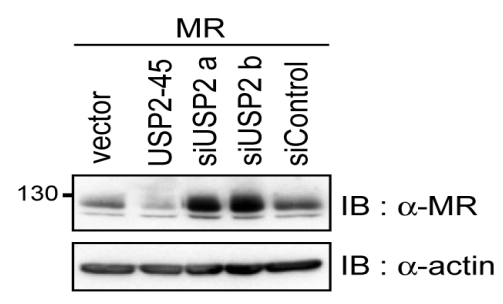

B

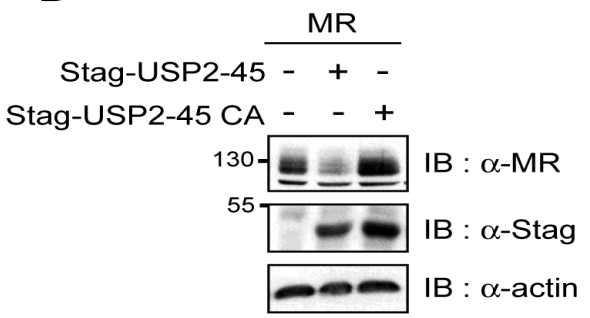

$\mathrm{D}$

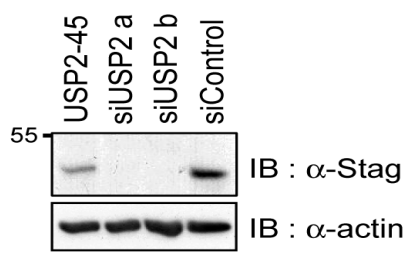

F

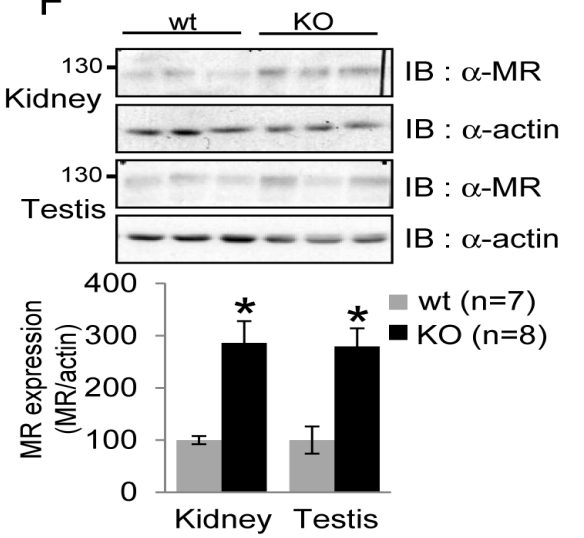

Fig. 3. USP2-45 decreases MR expression level. (A) M1 cells were co-transfected with MR and increasing doses of S-tagged USP2-45. The expression of proteins under investigation was determined by direct immunoblotting with anti-MR or anti-Stag antibodies and actin as a loading control. (B and C) M1 cells were co-transfected with MR with or without Stag-USP2-45 wt, Stag-USP2-45 CA (B) or USP2-69 (C). The expression of proteins under investigation was determined by direct immunoblotting with ant-MR, anti-Stag or actin as a loading control. (D) M1 cells were co-transfected with USP2-45 in presence or absence of siUSP2$45 \mathrm{a}$ or $\mathrm{b}$ and siControl. The efficiency of USP2-45 knock-down was evaluated by direct immunoblotting using anti-Stag and anti-actin as loading control. (E) M1 cells were co-transfected with MR, USP2-45, siUSP2-45a, siUSP2-45b or siControl. The expression of MR was determined by direct immunoblotting with anti-MR and anti-actin as loading control. (F) Kidney and testis lysates from wt or USP2 KO mice were submitted to immunoblotting using antibodies against MR and actin as a loading control. The MR expression level was quantified and values were normalized to actin and displayed as mean $\pm \operatorname{SEM}\left({ }^{*} \mathrm{p}<0.05\right)$.

life from approximately more than $4 \mathrm{~h}$ to $2.5 \mathrm{~h}$ (Fig. 4B). The decrease of MR protein level is mediated via proteasomal degradation since the proteasome blocker MG-132, but not the lysosome blocker chloroquine, limited the effect of USP2-45 on MR expression (Fig. 4C). In addition, we assessed the effects of USP2-45 on the state of MR polyubiquitylation. As shown in Figure 4D, wild-type USP2-45 increased the polyubiquitylated forms of MR, but not the catalytic inactive mutant. Taken together, these results suggest that the ubiquitin protease activity of USP2-45 is required for monoubiquitylation removal, as well as for subsequent polyubiquitylation and proteasomal degradation of MR. 


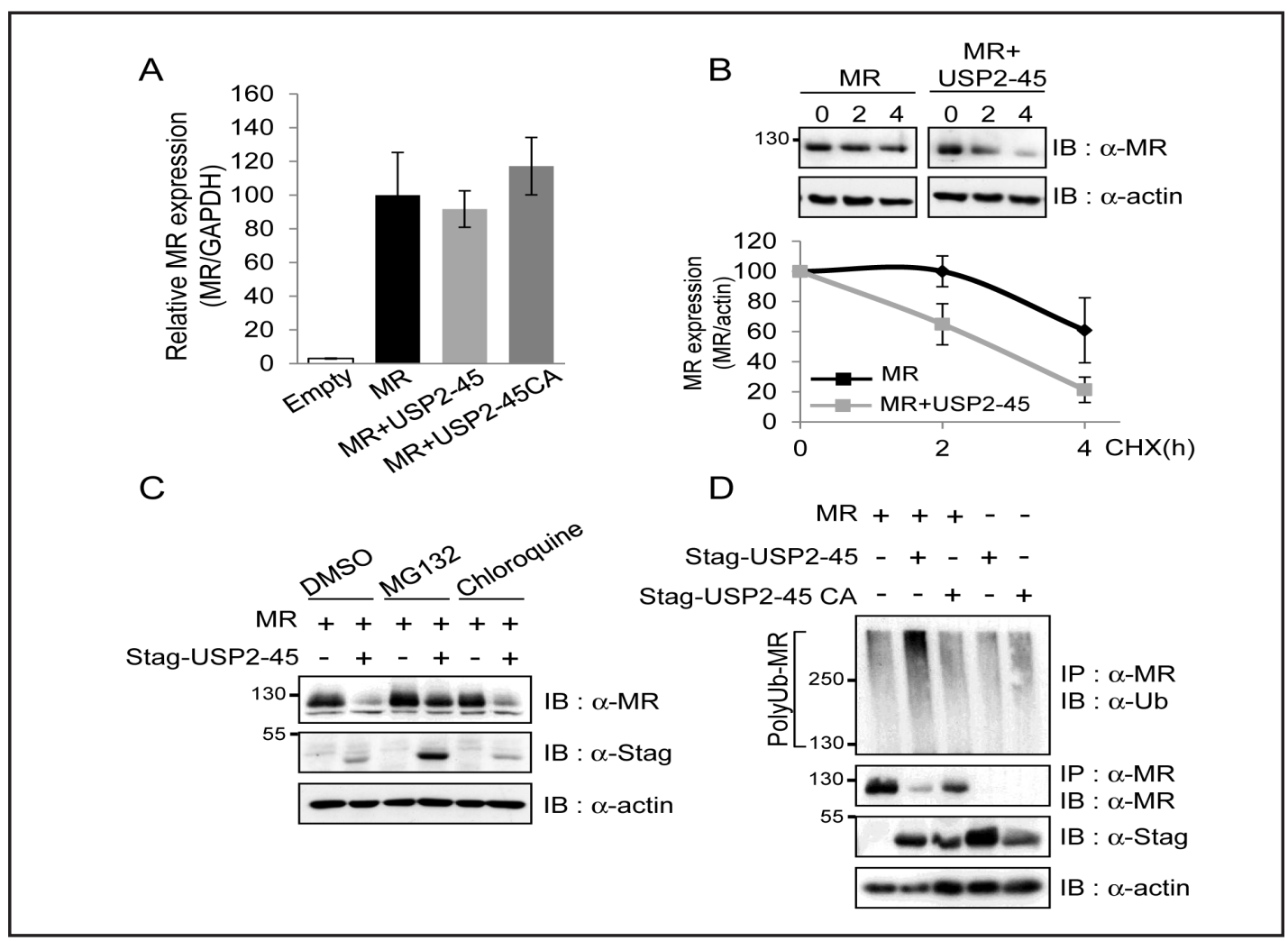

Fig. 4. USP2-45 decrease MR protein level via proteasomal degradation. (A) The abundance of MR mRNA in transfected M1 cells was measured by quantitative real-time reverse transcriptase-PCR (RT-PCR). Given are means relative to GAPDH \pm SEM ( $n=3$ ). (B) M1 cells were co-transfected with MR with or without of USP245. $24 \mathrm{~h}$ after transfection cells were treated with $10 \mu \mathrm{g} / \mathrm{ml}$ cycloheximide (CHX) for the indicated lengths of time. Cell lysates were subjected to immunoblotting with anti-MR antibody and actin as loading control. The MR expression level was quantified and values were normalized to actin and displayed as mean \pm SEM. (C) M1 cells were co-transfected with MR and Stag-USP2-45. The cells were treated for $6 \mathrm{~h}$ with $10 \mu \mathrm{M}$ MG132 before lysis. The expression of proteins under investigation was determined by direct immunoblotting and actin as a loading control. (D) M1 cells were co-transfected with MR and Stag-USP2-45 wt or CA. Cell lysates were immunoprecipitated with anti-MR and the ubiquitylated forms of MR were analyzed by immunoblotting with anti-Ub antibody or anti-MR antibody for immunoprecipitation control.

\section{USP2-45 limits aldosterone mediated transactivation}

To establish whether genomic responses to aldosterone were linked to USP2-45 expression, we first tested the effect of an increasing dose of USP2-45 on the aldosteroneresponsive reporter gene MMTV-luc. As shown in Figure 5A, USP2-45 repressed the basal as well as the aldosterone-dependent transcription in a dose dependent manner. This result could be related to the effect of USP2-45 on the cellular availability of MR. Conversely, depletion of USP2-45 by both shRNA targeted against USP2-45 increased the luciferase gene transactivation (Fig. 5B).

\section{Discussion}

Even if MR structure and function are still not well established as compared to other steroid receptors, intensive research on MR during the last decade indicated that its transcriptional activity requires different PTMs, such as ubiquitylation, sumoylation, acetylation or phosphorylation $[9,10,21]$. Moreover, the aldosterone/MR signaling pathway 


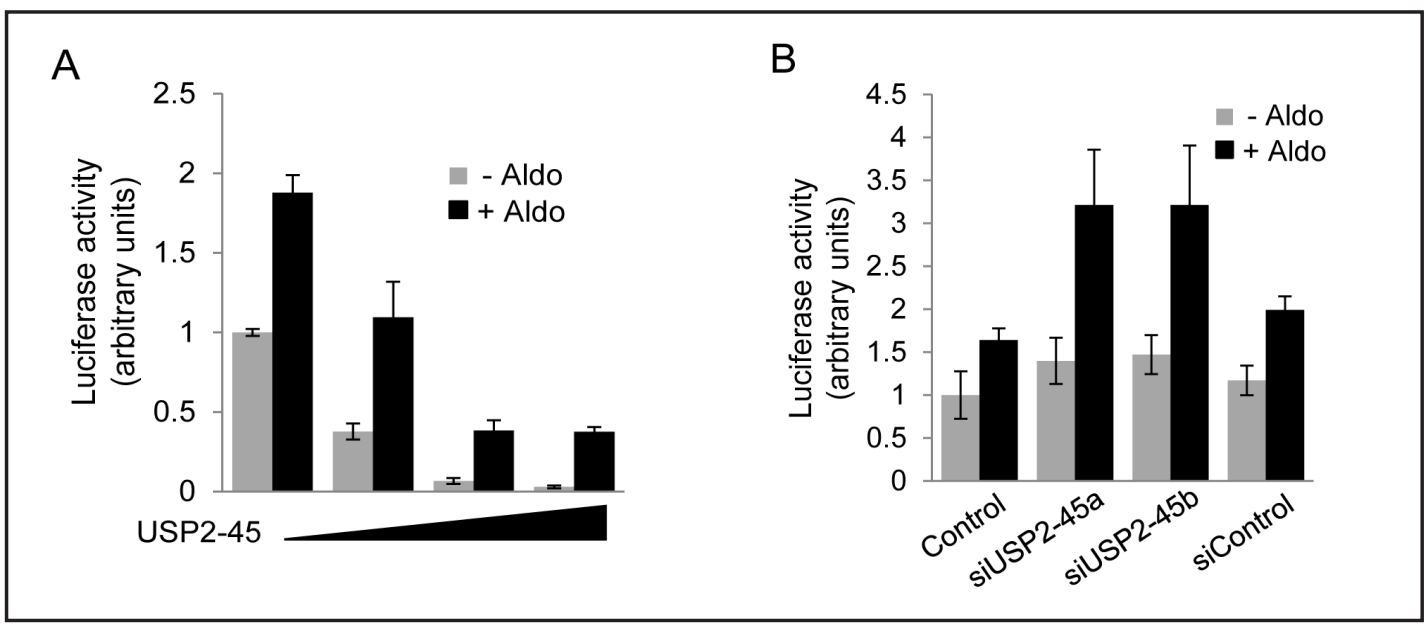

Fig. 5. USP2-45 limits aldosterone induced transcription. (A) M1 cells were transfected with MMTVluc, Stag-MR and an increasing dose of USP2-45. (B) M1 cells were transfected with MMTV-luc, Stag-MR, siUSP245a, siUSP2-45b or siControl. (A and B) Twenty-four hours after transfection, cells were treated for 16 hours with $30 \mathrm{nM}$ aldosterone. Subsequently, firefly luciferase (from MMTV-luc) and Renilla luciferase activity were measured. Data are given as mean \pm SD $(n=3)$.

requires the participation of numerous binding partners, which control aldosterone response in different target tissues. Several questions persist regarding reciprocal effects of these PTMs and the enzymes involved in such modifications.

Ubiquitylation is a PTM involving the sequential action of different enzymes targeting proteins for proteasomal degradation [11]. In the ubiquitin proteasome pathway, target proteins destined for proteasomal degradation are conjugated to polyubiquitin chains linked via ubiquitin lysine 48. However, other types of ubiquitylation (i.e. monoubiquitin, K63linked chains) are involved in non-canonical cellular processes independent of proteolytic degradation [22]. For example, monoubiquitylation might have several regulatory roles, such as changes in subcellular localization, activity, protein interactions, and protein stability [11, $22,23]$. Concerning the steroid receptors, it has been shown that ubiquitylation plays an important role in their activity as well as stability. Several of the enzymes involved in steroid receptor ubiquitylation have been identified (reviewed in [24]). However, for MR neither the enzymes, nor the precise residues where the modification occurs, were known.

Our study sheds light on the sequential events of MR ubiquitylation in renal epithelial cells. We propose a model in which basal monoubiquitylation of MR, which is sustained by the previously identified partner TSG101 [10], assures the receptor stability. We identified the aldosterone-induced protease USP2-45 as an interesting MR binding partner responsible for MR monoubiquitin removal (Fig. 6). Moreover, we provide evidence for the existence of a negative feedback loop that proceeds through the ability of USP2-45 to remove the monoubiquitin which facilitates the access of MR for ubiquitin-dependent degradation via the proteasome (Fig. 6). Other steroid receptors such as AR, GR or ER $\alpha$ have been shown to be monoubiquitylated [25-27], and this modification is regulated by their activation state. This study led us to propose that MR is regulated by a similar mechanism, even if the target residues or the enzymes involved are distinct.

Several lines of evidence support the concept that USP2-45 plays a crucial role in aldosterone-induced response. First, the aldosterone-induced protease USP2-45 can bind to non-activated MR in the cytoplasm. Second, over-expression of USP2-45 diminished MR expression level and depletion of endogenous USP2-45 stabilizes MR expression in vitro and in vivo, providing a molecular basis for the role of USP2-45 in promoting aldosterone/MR responses. Third, enforced expression of USP2-45 decreased the sensitivity of cells to aldosterone-induced transcription as evidenced by luciferase reporter genes. 
Fig. 6. Aldosterone-induced protease USP2-45 binds to MR in the cytoplasm and removing the monoubiquitin from MR that allows polyubiquitylation and degradation of the receptor via the proteasome.

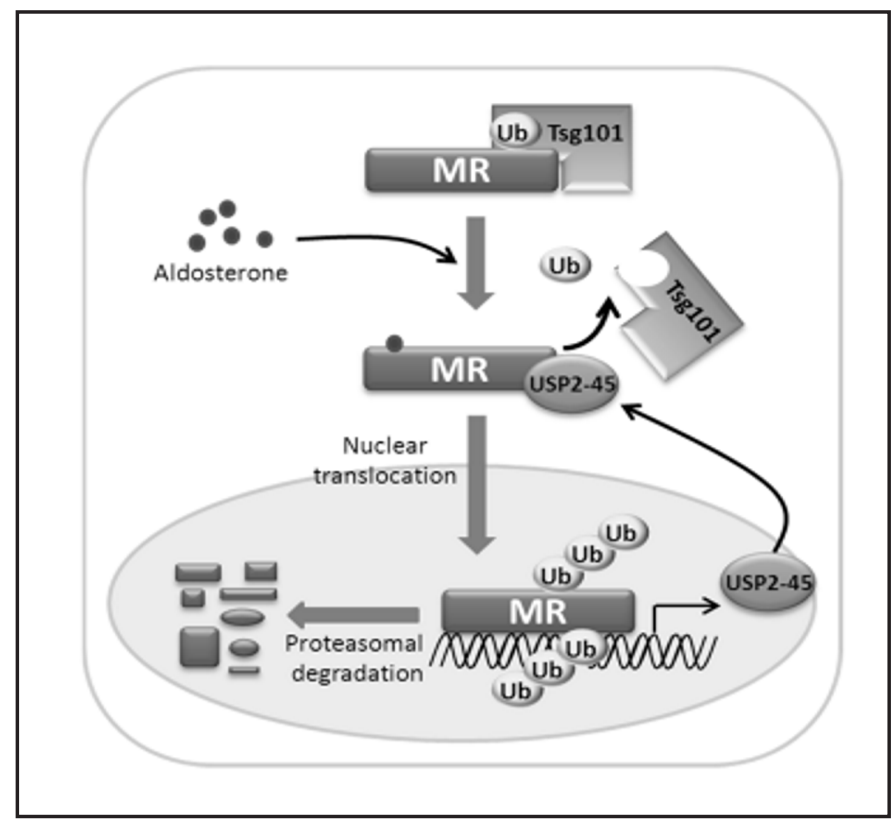

Conversely, depletion of USP2-45 enhanced the ability of aldosterone to induce target genes transactivation. Finally, we found that expression of USP2-45 decreased the formation of MR/ TSG101 complex, resulting in increased ubiquitylation of MR and its subsequent degradation through the proteasome. This suggests a new mechanism that allows quantitative clearance of MR protein in the presence of high levels of aldosterone hormone. Alternatively, USP2-45 may limit the availability of MR to set either a threshold level or a ceiling for aldosteronemediated cell response, depending on cell types, the level of expressed USP2-45, or the intensity of hormonal activation.

USP2 has been studied in vivo in two different constitutive knockout models. In the first model, USP 2 was inactivated by deleting exons 4 to 6 encoding the catalytic domain [28]. These mice are viable, and no renal dysfunction linked to an apparent mineralocorticoid excess were described. However, male mice lacking Usp2 gene showed a severe defect in fertility, even if spermatogenesis was not significantly altered. The mechanisms involved in this phenotype are still unclear. Nevertheless, it is known that USP2-45 and MR are highly expressed in the testis $[15,29]$, and we found that MR expression was also significantly higher in testis of USP2 null mice. This overexpression could be one explanation for UPS2 null mice infertility, since a direct correlation was described between adrenal hyper-secretion of MR ligands (aldosterone and cortisol) and the proportion of spermatozoa with abnormal headpiece structure in the human ejaculate [30]. These observations suggested a cause-andeffect relationship between MR activity and infertility.

A second USP2 KO model was generated by deleting exons 3 and 4, which encode a portion of the catalytic region [31]. These mice lacking the deubiquitylating enzyme USP2 are hypersensitive to light at the day-to-night transition, resulting in enhanced phase delays. This phenotype explanation by authors was based on a model in which the circadian machinery was disturbed, particularly expression of the BMAL1 transcription factor. The higher turnover of BMAL1 protein in USP2 KO mice could account for the altered expression of clock controlled genes and core clock components [31]. In this context, the hypersensitivity to light might be due to an altered response of the SCN to light input or further upstream within the retina. In addition to USP2-45, MR is also well expressed in the inner retina and is crucial for the photoreceptor function through a tight regulation of ion/water channels expression [32-34]. It is therefore possible that increased MR expression in USP2 KO mice is the cause for their light hypersensitivity via photoreceptors over-activity.

In summary, our study provides the basis for an original mechanism of aldosterone/ MR signaling regulation based on USP2-45. We propose a novel role for USP2-45 as a 
central regulator of MR, able to remove the receptor monoubiquitylation and induced its destabilization. Future studies are required to identify the lysine residue where the monoubiquitylation of MR occurs, as well as the ubiquitin ligase(s) involved in mono- or polyubiquitylation and subsequent degradation of this receptor.

\section{Acknowledgements}

We thank Dr. R. D. Rajaram for critically reading the manuscript. We also thank D. Pouly for the tissue lysate preparation from USP2 knock-out mice, Dr. P. Shaw (Lausanne) for providing an HA-ubiquitin encoding plasmid and P.J. Fuller for the MMTV-luc plasmid. This work was supported by the Leducq Foundation (Transatlantic Network on Hypertension), Swiss National Science Foundation (31003A_125422/1), the Swiss NCCR Kidney.ch and an EMBO long-term fellowship to NF.

\section{References}

$>1$ Arriza JL, Weinberger C, Cerelli G, Glaser TM, Handelin BL, Housman DE, Evans RM: Cloning of human mineralocorticoid receptor complementary DNA: Structural and functional kinship with the glucocorticoid receptor. Science 1987;237:268-275.

2 Staub 0, Loffing J: Mineralocorticoid action in the aldosterone sensitive distal nephron; in Alpern RJ, Caplan MJ, Moe OW (eds): The kidney: Physiology and pathophysiology. London, Waltham, San Diego, Adademic Press, 2013, vol 1, pp 1181-1211.

-3 Geller DS, Rodriguezsoriano J, Boado AV, Schifter S, Bayer M, Chang SS, Lifton RP: Mutations in the mineralocorticoid receptor gene cause autosomal dominant pseudohypoaldosteronism type i. Nat Genet 1998;19:279-281.

-4 Geller DS, Farhi A, Pinkerton N, Fradley M, Moritz M, Spitzer A, Meinke G, Tsai FT, Sigler PB, Lifton RP: Activating mineralocorticoid receptor mutation in hypertension exacerbated by pregnancy. Science 2000;289:119-123.

5 Shibata S, Fujita T: Mineralocorticoid receptors in the pathophysiology of chronic kidney diseases and the metabolic syndrome. Mol Cell Endocrinol 2012;350:273-280.

-6 Pitt B, Reichek N, Willenbrock R, Zannad F, Phillips RA, Roniker B, Kleiman J, Krause S, Burns D, Williams GH: Effects of eplerenone, enalapril, and eplerenone/enalapril in patients with essential hypertension and left ventricular hypertrophy: The 4e-left ventricular hypertrophy study. Circulation 2003;108:1831-1838. Pitt B, Remme W, Zannad F, Neaton J, Martinez F, Roniker B, Bittman R, Hurley S, Kleiman J, Gatlin M: Eplerenone, a selective aldosterone blocker, in patients with left ventricular dysfunction after myocardial infarction. N Engl J Med 2003;348:1309-1321.

-8 Pitt B, Zannad F, Remme WJ, Cody R, Castaigne A, Perez A, Palensky J, Wittes J: The effect of spironolactone on morbidity and mortality in patients with severe heart failure. Randomized aldactone evaluation study investigators. N Engl J Med 1999;341:709-717.

-9 Viengchareun S, Le Menuet D, Martinerie L, Munier M, Pascual-Le Tallec L, Lombes M: The mineralocorticoid receptor: Insights into its molecular and (patho)physiological biology. Nucl Recept Signal 2007;5:e012.

10 Faresse N, Vitagliano JJ, Staub 0: Differential ubiquitylation of the mineralocorticoid receptor is regulated by phosphorylation. FASEB J 2012;26:4373-4382.

11 Weissman AM, Shabek N, Ciechanover A: The predator becomes the prey: Regulating the ubiquitin system by ubiquitylation and degradation. Nat Rev Mol Cell Biol 2011;12:605-620.

12 Komander D, Clague MJ, Urbe S: Breaking the chains: Structure and function of the deubiquitinases. Nat Rev Mol Cell Biol 2009;10:550-563.

-13 Nijman SM, Luna-Vargas MP, Velds A, Brummelkamp TR, Dirac AM, Sixma TK, Bernards R: A genomic and functional inventory of deubiquitinating enzymes. Cell 2005;123:773-786. 
Faresse/Debonneville/Staub: Deubiquitylation Induces MR Degradation

14 Lin H, Keriel A, Morales CR, Bedard N, Zhao Q, Hingamp P, Lefrancois S, Combaret L, Wing SS: Divergent n-terminal sequences target an inducible testis deubiquitinating enzyme to distinct subcellular structures. Mol Cell Biol 2000;20:6568-6578.

15 Gousseva N, Baker RT: Gene structure, alternate splicing, tissue distribution, cellular localization, and developmental expression pattern of mouse deubiquitinating enzyme isoforms usp2-45 and usp2-69. Gene Expr 2003;11:163-179.

-16 Fakitsas P, Adam G, Daidie D, van Bemmelen MX, Fouladkou F, Patrignani A, Wagner U, Warth R, Camargo SM, Staub O, Verrey F: Early aldosterone-induced gene product regulates the epithelial sodium channel by deubiquitylation. J Am Soc Nephrol 2007;18:1084-1092.

17 Ruffieux-Daidie D, Poirot O, Boulkroun S, Verrey F, Kellenberger S, Staub O: Deubiquitylation regulates activation and proteolytic cleavage of enac. J Am Soc Nephrol 2008;19:2170-2180.

18 Gomez-Sanchez CE, de Rodriguez AF, Romero DG, Estess J, Warden MP, Gomez-Sanchez MT, GomezSanchez EP: Development of a panel of monoclonal antibodies against the mineralocorticoid receptor. Endocrinology 2006;147:1343-1348.

19 Faresse N, Ruffieux-Daidie D, Salamin M, Gomez-Sanchez CE, Staub O: Mineralocorticoid receptor degradation is promoted by hsp90 inhibition and the ubiquitin-protein ligase chip. Am J Physiol Renal Physiol 2010;299:F1462-1472.

20 Rogerson FM, Dimopoulos N, Sluka P, Chu S, Curtis AJ, Fuller PJ: Structural determinants of aldosterone binding selectivity in the mineralocorticoid receptor. J Biol Chem 1999;274:36305-36311.

-21 Tallec LP, Kirsh O, Lecomte MC, Viengchareun S, Zennaro MC, Dejean A, Lombes M: Protein inhibitor of activated signal transducer and activator of transcription 1 interacts with the n-terminal domain of mineralocorticoid receptor and represses its transcriptional activity: Implication of small ubiquitin-related modifier 1 modification. Mol Endocrinol 2003;17:2529-2542.

-22 Haglund K, Dikic I: Ubiquitylation and cell signaling. EMBO J 2005;24:3353-3359.

23 Staub 0, Rotin D: Role of ubiquitylation in cellular membrane transport. Physiol Rev 2006;86:669-707.

24 Faus H, Haendler B: Post-translational modifications of steroid receptors. Biomed Pharmacother 2006;60:520-528.

25 Ma Y, Fan S, Hu C, Meng Q, Fuqua SA, Pestell RG, Tomita YA, Rosen EM: Brca1 regulates acetylation and ubiquitination of estrogen receptor-alpha. Mol Endocrinol 2010;24:76-90.

26 Burgdorf S, Leister P, Scheidtmann KH: Tsg101 interacts with apoptosis-antagonizing transcription factor and enhances androgen receptor-mediated transcription by promoting its monoubiquitination. J Biol Chem 2004;279:17524-17534.

27 Ismaili N, Blind R, Garabedian MJ: Stabilization of the unliganded glucocorticoid receptor by tsg101. J Biol Chem 2005;280:11120-11126.

28 Bedard N, Yang Y, Gregory M, Cyr DG, Suzuki J, Yu X, Chian RC, Hermo L, O'Flaherty C, Smith CE, Clarke HJ, Wing SS: Mice lacking the usp2 deubiquitinating enzyme have severe male subfertility associated with defects in fertilization and sperm motility. Biol Reprod 2011;85:594-604.

29 Le Menuet D, Viengchareun S, Penfornis P, Walker F, Zennaro MC, Lombes M: Targeted oncogenesis reveals a distinct tissue-specific utilization of alternative promoters of the human mineralocorticoid receptor gene in transgenic mice. J Biol Chem 2000;275:7878-7886.

30 Mazo EB, Koriakin MV, Kudriavtsev Iu V, Evseev LP, Akopian AS: [The role of disorders of mineralocorticoid function of the adrenal glands in the development of infertility in patients with left-side varicocele]. Urol Nefrol (Mosk) 1989;2:38-45.

31 Scoma HD, Humby M, Yadav G, Zhang Q Fogerty J, Besharse JC: The de-ubiquitinylating enzyme, usp2, is associated with the circadian clockwork and regulates its sensitivity to light. PLoS One 2011;6:e25382.

-32 Mirshahi M, Nicolas C, Mirshahi A, Hecquet C, d'Hermies F, Faure JP, Agarwal MK: The mineralocorticoid hormone receptor and action in the eye. Biochem Biophys Res Commun 1996;219:150-156.

33 Golestaneh N, Picaud S, Mirshahi M: The mineralocorticoid receptor in rodent retina: Ontogeny and molecular identity. Mol Vis 2002;8:221-225.

34 Zhao M, Valamanesh F, Celerier I, Savoldelli M, Jonet L, Jeanny JC, Jaisser F, Farman N, Behar-Cohen F: The neuroretina is a novel mineralocorticoid target: Aldosterone up-regulates ion and water channels in muller glial cells. FASEB J 2010;24:3405-3415. 